\title{
Total Synthesis of (-)-Isoavenaciolide
}

David Santos, Xavier Ariza, * Jordi Garcia,* Paul Lloyd-Williams, Agustín Martínez-Laporta, and

Carolina Sánchez

Departament de Química Orgànica and Institut de Biomedicina de la Universitat de Barcelona (IBUB),

Facultat de Química, Universitat de Barcelona, Martí i Franquès 1, 08028-Barcelona, Spain

xariza@ub.edu,jordigarciagomez@ub.edu

\section{RECEIVED DATE}

TITLE RUNNING HEAD: Total synthesis of (-)-Isoavenaciolide

CORRESPONDING AUTHOR FOOTNOTE: Corresponding author. Tel.: +34 934039114; fax: +34 933397878.

ABSTRACT: An enantioselective approach to (-)-isoavenaciolide was achieved starting from 1undecyn-3-ol. The synthesis relied upon the preparation of a chiral 4-silyloxy-2-alkenylborane by hydroboration of a protected 2,3-allenol and subsequent stereoselective addition to 2thiophenecarboxaldehyde.

KEYWORDS. Allene, hydroboration, natural product, total synthesis, asymmetric synthesis

\section{Introduction}

Isoavenaciolide ((-)-1) is a member of a distinct family of $\alpha$-methylene-bis(butyrolactones) natural products isolated from the fermentation broth of Aspergillus and Penicillium species. ${ }^{1}$ This secondary 
metabolite displays a broad spectrum of antibacterial and antifungal properties and inhibits vaccinia $\mathrm{H} 1$ related (VHR) phosphatase activity (Figure 1$).^{2}$

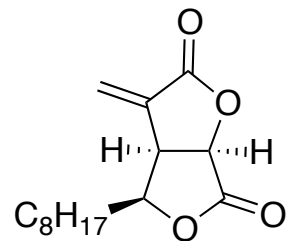

$(-)-1$

Isoavenaciolide<smiles>C=C1C(=O)O[C@H]2C(=O)O[C@@H]([12F])[C@@H]12</smiles>

Avenaciolide<smiles>C=C1C(=O)O[C@H]2C(=O)O[C@@H](CC)[C@H]12</smiles>

Ethisolide

Figure 1. Isoavenaciolide and other related natural $\alpha$-methylene-bislactones

On account of its biological activity and its interesting bislactone skeleton numerous enantioselective synthesis have been reported. ${ }^{3}$ Most of the initial approaches relied either on the transformation of chiral natural products ${ }^{4}$ or on the Sharpless epoxidation..$^{5}$ Only recently have other stereoselective methods been used to synthesize this molecule. ${ }^{6}$ In our search for new approaches to the preparation of polyhydroxylated frameworks we have developed a stereoselective method for the preparation of 1,3diols based on a tandem process that involves hydroboration of a chiral protected 2,3-allenol followed by addition of an aldehyde. ${ }^{7}$ We anticipated that this methodology could be applied to the synthesis of (-)-isoavenaciolide as a representative example of this family of compounds. In our retrosynthetic analysis of (-)-1, the methylene group would be introduced in the last step from bislactone $\mathbf{2}$ that would arise from dihydroxy diacid 3. Such a structure could be prepared by a double oxidation of a homoallylic diol that can be synthesized stereoselectively with our methodology (Scheme 1). ${ }^{8}$ 


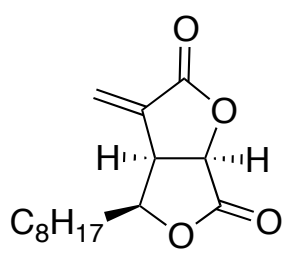

$(-)-1$

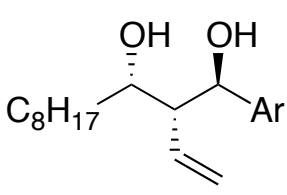

$\sqrt{ }$

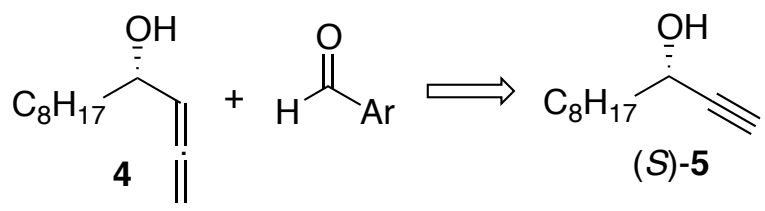

Scheme 1. Retrosynthetic analysis of (-)-1.

\section{Results and Discussion}

The synthesis was initiated by preparation of the enantioenriched allenol 4. 2,3-Allenols can be easily obtained from the corresponding propargylic alcohols by a $\mathrm{Cu}(\mathrm{I})$-mediated homologation process with paraformaldehyde. ${ }^{9}$ Among the variety of methods available for the synthesis of enantiopure 1-alkyn-3ols such as $(S)-5$, we preferred to employ one based on enzymatic resolution. ${ }^{10}$ Thus, kinetic resolution of 1-undecyn-3-ol ( \pm )-(5) with Novozym 435 (Candida antarctica lipase) and vinyl acetate afforded enantioenriched $(S)-\mathbf{5}$ as acetate $\mathbf{6}$ that was hydrolyzed and homologated to allenol 4 under the conditions described above (Scheme 2). ${ }^{11}$ 


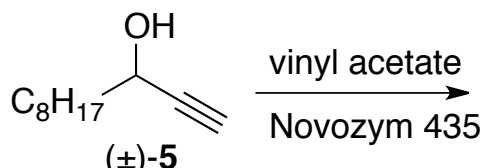

$( \pm)-5$

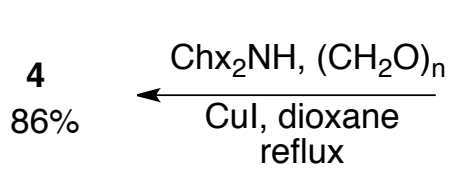

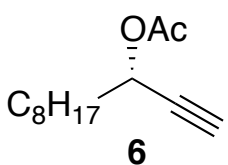

$35 \%$

$\mathrm{NaOMe}$

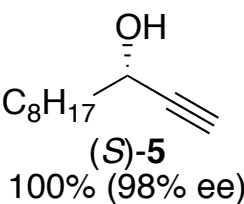

Scheme 2. Preparation of enantioenriched allenol 4

Our recently-described methodology of addition of protected 2,3-allenols to aldehydes is based on the hydroboration of an allene and the addition of the transient 2-alkenylborane to an aldehyde (Scheme 3). Initially, the borane adds to the sterically less hindered face of the allene to form a ( $Z$ )-2-alkenylborane. The addition of an aromatic aldehyde to this then affords a syn,anti homoallylic alcohol through a 6membered transition state. The anti relationship between the vinyl and hydroxyl groups arises from the stereochemistry of the olefin $(Z)$ whereas the syn relationship of the vinyl and the silyloxy groups derives from the face of the aldehyde that is added to the chiral 2-alkenylborane. An important feature of our method is that the kinetically formed $(Z)$-borane isomerizes to the thermodynamically more stable (E)-2-alkenylborane at room temperature, such that when the aldehyde is not added immediately, isomerization can occur and the syn,syn stereoisomer is obtained as the major product. Consequently, the syn,anti stereoisomer is only obtained as the major isomer when an aromatic aldehyde is employed and the ( $Z$ )-borane is trapped before isomerization. 


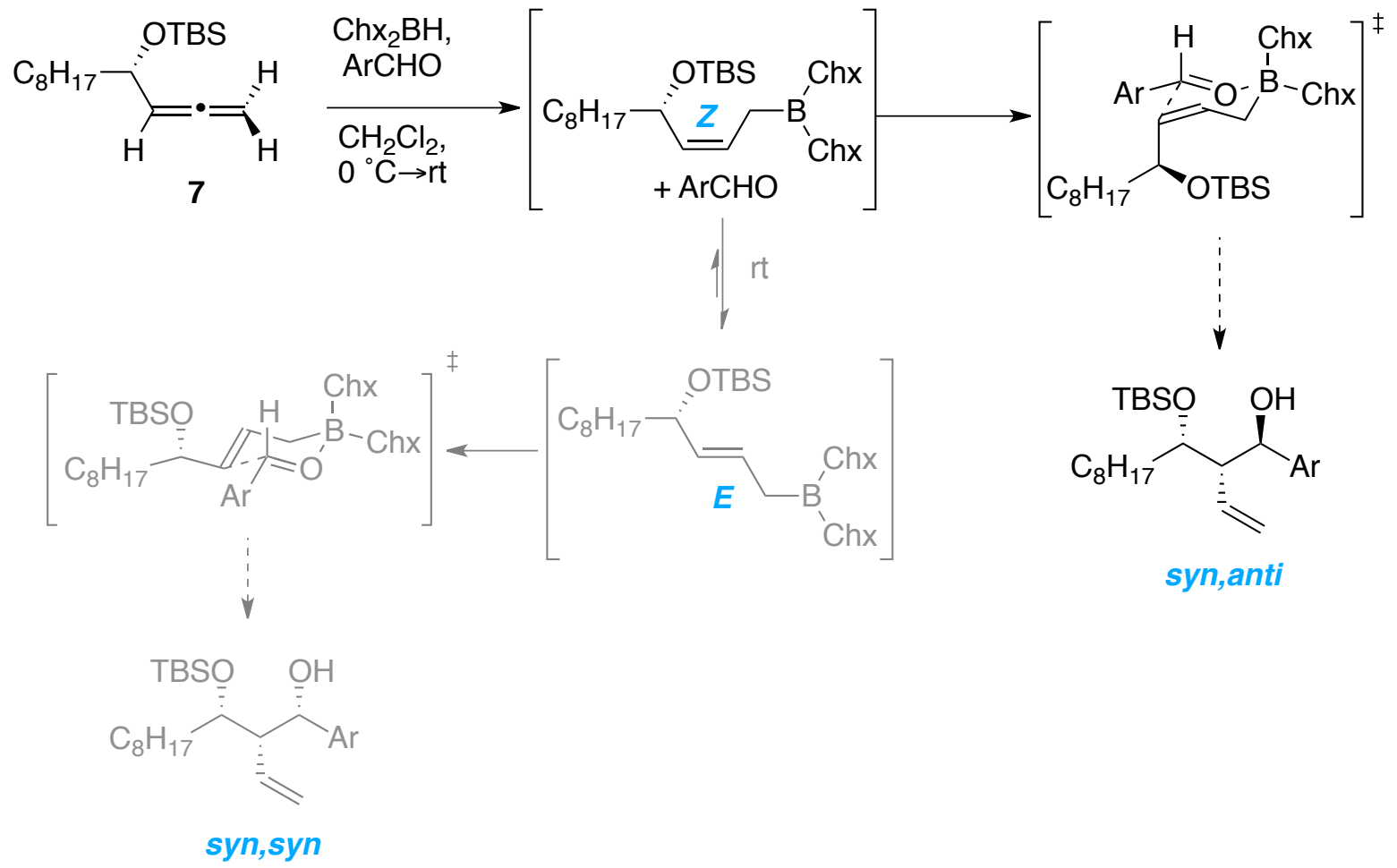

Scheme 3. Addition of protected 2,3-allenols to aldehydes

In the case in hand, since the required stereochemistry was syn,anti (Scheme 2), an aromatic aldehyde was required in order to ensure high stereoselectivities. ${ }^{12}$ Among the different possibilities, we chose 2thiophenecarboxaldehyde on account of its being easier to oxidize at a later stage in the synthesis. In previous studies, ${ }^{7}$ we have shown that the TBS group is a very convenient option for the protection of 4 in these additions, whereas other silicon-based protecting groups such as TBDPS lowered the stereoselectivity of the addition. Thus, allene 7 was prepared by protection of allenol 4 with TBSchloride (Scheme 4), and its addition to 2-thiophenecarboxaldehyde gave a diastereomeric mixture ( $\mathrm{dr}$ 84:16) of syn,anti-8 and syn,syn-8. The expected major isomer syn,anti-8 was isolated in $80 \%$ yield. $^{13}$ 


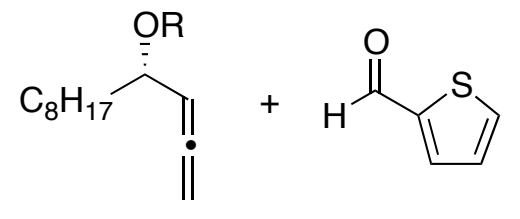

$4(\mathrm{R}=\mathrm{H})$

7 (R=TBS, 89\%) $\longleftarrow$ imidazole
1) $\mathrm{Chx}_{2} \mathrm{BH}$

2) $\underset{\mathrm{N}\left(\mathrm{CH}_{2} \mathrm{CH}_{2} \mathrm{OH}\right)_{3}}{\stackrel{\mathrm{CH}_{2} \mathrm{Cl}_{2}, \mathrm{rt} \rightarrow 0{ }^{\circ} \mathrm{C}}{\longrightarrow}}$

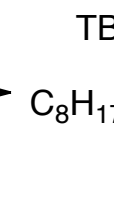

$\mathrm{C}_{8} \mathrm{H}_{17}$

(1)

$80 \%$

syn,anti-8

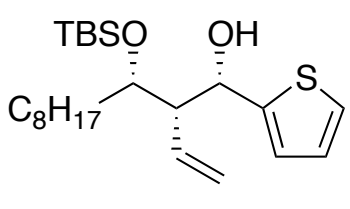

(dr 84:16)

syn, syn-8

Scheme 4. Addition of allene 7 to 2-thiophenecarboxaldehyde

The oxidation of the terminal olefin in syn,anti-8 to a carboxylic acid was planned to take place in two steps: initial regioselective oxidation of the vinyl group to the primary alcohol followed by concomitant oxidation ${ }^{14}$ of this and the thiophene with $\mathrm{NaIO}_{4} / \mathrm{RuCl}_{3}$ which would afford dicarboxylic acid 3 .

An expeditious method for achieving this turned out to be protection of both oxygens of syn,anti-8 as acetyl groups (Scheme 5). Thus deprotection of the TBS group of $\mathbf{8}$ afforded diol $\mathbf{9}$ and its acetylation gave diacetylated olefin 10. This was then hydroborated with dicyclohexylborane and the resulting borane was oxidized at neutral $\mathrm{pH}$ to afford 11. Simultaneous oxidation of the alcohol and the thiophene moiety then afforded dicarboxylic acid 12 in good yield. Nevertheless, hydroboration/oxidation of syn,anti-8 did require care in its execution. Basic oxidations of the borane intermediate (with $\mathrm{H}_{2} \mathrm{O}_{2} / \mathrm{NaOH}$ ) promoted the migration of an acetyl group to the primary alcohol of $\mathbf{1 1}$ and crude $\mathbf{1 1}$ required immediate purification in order to avoid its decomposition. Protective group migration could not be avoided by switching to temporary silicon-based groups such as TBS or TBDPS nor by using other hydroborating systems such as $\mathrm{BH}_{3}: \mathrm{SMe}_{2}$ or catecholborane/Rh (Table 1). Neither were yields of 11 improved using these reagents. 


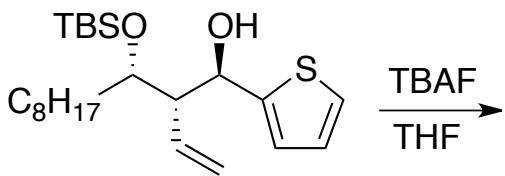

syn,anti-8

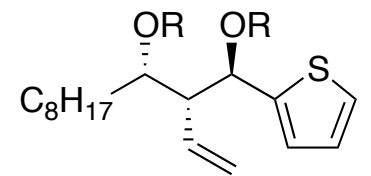

$9(\mathrm{R}=\mathrm{H}, 87 \%)$
$10(\mathrm{R}=\mathrm{Ac}, 97 \%)$$\longrightarrow \mathrm{Ac}_{2} \mathrm{O}$

1) $\mathrm{Chx}_{2} \mathrm{BH}$

2) $\mathrm{H}_{2} \mathrm{O}_{2}$, buffer $\mathrm{pH}=7$

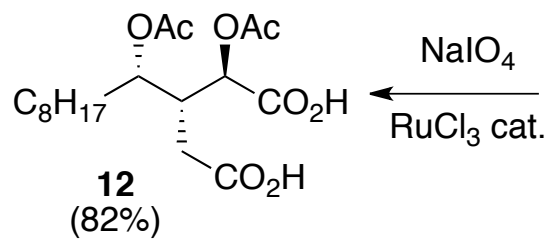<smiles>CC(=O)O[C@H]([18O]C(C)=O)[C@@H](CCO)[C@H](CCO)c1cccs1</smiles>

Scheme 5. Synthesis of dicarboxylic acid 12.

Table 1. Hydroboration of $\mathbf{1 0 .}$

\begin{tabular}{|l|l|l|l|}
\hline Entry & Hydroborating agent & Oxidant & Yield(\%) \\
\hline 1 & Catecholborane/RhCl($\left(\mathrm{PPh}_{3}\right)_{3}$ & $\mathrm{H}_{2} \mathrm{O}_{2} / \mathrm{NaOH}$ & 0 \\
\hline 2 & Catecholborane/ $\mathrm{RhCl}\left(\mathrm{PPh}_{3}\right)_{3}$ & $\mathrm{H}_{2} \mathrm{O}_{2} /$ buffer $\mathrm{pH}=7$ & 40 \\
\hline 3 & $\mathrm{BH}_{3}: \mathrm{SMe}_{2}$ & $\mathrm{H}_{2} \mathrm{O}_{2} /$ buffer $\mathrm{pH}=7$ & 0 \\
\hline 4 & $\mathrm{BH}_{3}: \mathrm{SMe}_{2}$ & $\mathrm{H}_{2} \mathrm{O}_{2} / \mathrm{NaOH}$ & 26 \\
\hline 5 & $\mathrm{Chx}_{2} \mathrm{BH}$ & $\mathrm{H}_{2} \mathrm{O}_{2} /$ buffer $\mathrm{pH}=7$ & 87 \\
\hline
\end{tabular}

The final steps of the synthesis were quite straightforward. Hydrolysis of crude diacetate $\mathbf{1 2}$ afforded dihydroxy diacid $\mathbf{3}$ that cyclized in situ giving bislactone $\mathbf{2}$. Methylenation was easily achieved by a known procedure ${ }^{15}$ that completed the total synthesis of (-)-isoavenaciolide. The optical rotation of the synthetic product was in good agreement with the value reported in the literature. ${ }^{5 \mathrm{~d}}$ 


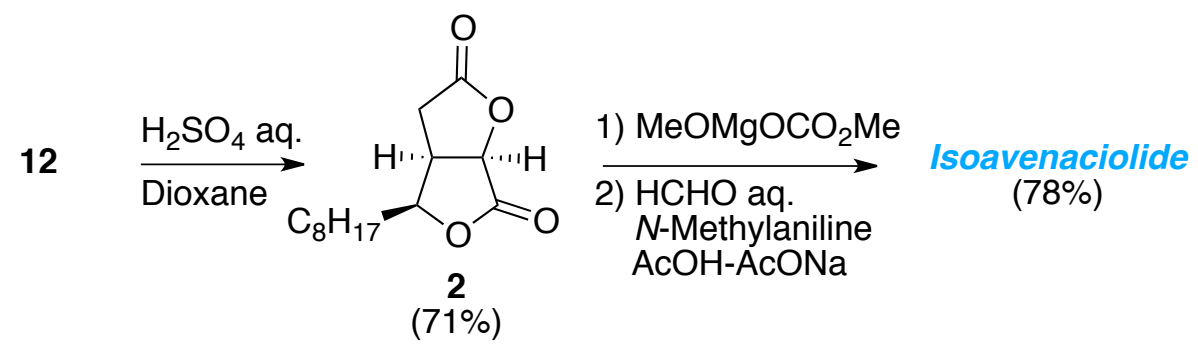

Scheme 6. Final steps towards (-)-isoavenaciolide.

\section{Conclusions}

The enantioselective synthesis of (-)-isoavenaciolide (1) described here constitutes a direct application of our recent stereodivergent approach to 2-vinyl-1,3-diols based on a tandem allene hydroboration/aldehyde addition process to natural product synthesis. This approach takes advantage of the good facial discrimination of aromatic aldehydes by the transient chiral (Z)-2-alkenylborane formed from a chiral allene. Temporary protection of 1,3-diol $\mathbf{9}$ as its diacetate $\mathbf{1 0}$ very conveniently facilitated the oxidation steps that led to diacid $\mathbf{1 2}$ that then cyclized to bislactone $\mathbf{2}$.

\section{Experimental Section}

All reactions involving moisture- or air-sensitive reagents were performed in oven-dried glassware under $\mathrm{N}_{2}$. Chemical shifts $(\delta)$ are quoted in parts per million and referenced to internal TMS for ${ }^{1} \mathrm{H}$ NMR and to $\mathrm{CDCl}_{3}(\delta 77.0 \mathrm{ppm})$ or $\mathrm{CD}_{3} \mathrm{OD}(\delta 49.0 \mathrm{ppm})$ for ${ }^{13} \mathrm{C}$ NMR. Column chromatography was performed on silica gel (Merck 230-400 mesh). HRMS analyses were recorded on a LC/MSD-TOF mass spectrometer.

( \pm )-Undec-1-yn-3-ol (( \pm )-5). $n$-Butyllithium $(2.5 \mathrm{M}$ in hexanes, $13.2 \mathrm{~mL}, 33 \mathrm{mmol})$ was added to a solution of ethynyltrimethylsilane $(4.57 \mathrm{~mL}, 33 \mathrm{mmol})$ in anhydrous THF under $\mathrm{N}_{2}$ at $-40{ }^{\circ} \mathrm{C}$. The mixture was stirred for $10 \mathrm{~min}$ and nonanal $(5.15 \mathrm{~mL}, 30 \mathrm{mmol})$ was added dropwise at $-40{ }^{\circ} \mathrm{C}$. After 10 min the reaction was allowed to warm to rt and then stirred for $45 \mathrm{~min}$. The reaction was quenched with $\mathrm{H}_{2} \mathrm{O}(20 \mathrm{~mL})$ and $\mathrm{K}_{2} \mathrm{CO}_{3}(2.5 \mathrm{~g})$ and stirred for $2 \mathrm{~h}$. The aqueous layer was extracted with $\mathrm{CH}_{2} \mathrm{Cl}_{2}(3 \times 10$ $\mathrm{mL}$ ), the organic layer was dried over $\mathrm{MgSO}_{4}$ and solvents were removed. Flash chromatography (silica gel, hexanes/AcOEt 98:2) gave ( \pm )-5 as a colorless oil (4.64 g, $27.6 \mathrm{mmol}, 92 \%): R_{f}$ (hexanes/AcOEt 
8:2): 0.58; ${ }^{1} \mathrm{H}$ NMR $\left(\mathrm{CDCl}_{3}, 400 \mathrm{MHz}\right): \delta 0.87(3 \mathrm{H}, \mathrm{t}, J=6.8 \mathrm{~Hz}), 1.25-1.35(10 \mathrm{H}, \mathrm{m}), 1.40-1.50(2 \mathrm{H}$, m), 1.67-1.75 (2H, m), $1.80(1 \mathrm{H}, \mathrm{bs}), 2.45(1 \mathrm{H}, \mathrm{d}, J=3.0 \mathrm{~Hz}), 4.36(1 \mathrm{H}, \mathrm{td}, J=6.4,3.0 \mathrm{~Hz}) ;{ }^{13} \mathrm{C} \mathrm{NMR}$ $\left(\mathrm{CDCl}_{3}, 100 \mathrm{MHz}\right): \delta 14.1,22.6,25.0,29.2,29.2,29.5,31.8,37.7,62.3,72.8,85.0$; IR (film, $\left.\mathrm{cm}^{-1}\right)$ : 3406, 3302, 2928, 2157, 1098; HRMS (ESI+): calcd for $\mathrm{C}_{11} \mathrm{H}_{21} \mathrm{O}(\mathrm{M}+\mathrm{H})^{+}$169.1587, found 169.1592.

(S)-Undec-1-yn-3-yl acetate (6). Racemic alcohol ( \pm )-5 (4.64 g, $27.6 \mathrm{mmol})$ was treated with vinyl acetate $(30 \mathrm{~mL})$ in the presence of Novozym $435(0.250 \mathrm{~g})$. The mixture was stirred under $\mathrm{N}_{2}$, until ${ }^{1} \mathrm{H}$ NMR showed $40 \%$ conversion. The mixture was filtered and the solvent removed. The crude product was purified by flash chromatography (silica gel, hexanes/AcOEt 9:1) to give 5 (2.704 g, $16.1 \mathrm{mmol}$, $58 \%)$ and (-)-6 (2.05 g, $9.8 \mathrm{mmol}, 35 \%)$ as a colorless oil; $R_{f}\left(\right.$ hexanes/AcOEt 8:2): $0.83 ;[\alpha]_{\mathrm{D}}{ }^{25}-58.6(c$ 0.99, $\left.\mathrm{CHCl}_{3}\right) ;{ }^{1} \mathrm{H} \mathrm{NMR}\left(\mathrm{CDCl}_{3}, 300 \mathrm{MHz}\right): \delta 0.88(3 \mathrm{H}, \mathrm{t}, J=6.8 \mathrm{~Hz}), 1.25-1.35(10 \mathrm{H}, \mathrm{m}), 1.39-1.48$ $(2 \mathrm{H}, \mathrm{m}), 1.73-1.80(2 \mathrm{H}, \mathrm{m}), 2.09(3 \mathrm{H}, \mathrm{s}), 2.44(1 \mathrm{H}, \mathrm{d}, J=2.2 \mathrm{~Hz}), 5.33(1 \mathrm{H}, \mathrm{td}, J=6.9,2.2 \mathrm{~Hz}) ;{ }^{13} \mathrm{C}$ NMR $\left(\mathrm{CDCl}_{3}, 75 \mathrm{MHz}\right): \delta 14.1,21.0,22.6,24.9,29.1,29.2,29.4,31.8,34.6,63.8,73.3,81.3,169.9$; IR (film, $\left.\mathrm{cm}^{-1}\right): 3311,2924,2166,1740,1226$; HRMS (ESI+): calcd for $\mathrm{C}_{13} \mathrm{H}_{22} \mathrm{NaO}_{2}(\mathrm{M}+\mathrm{Na})^{+} 233.1512$, found 233.1519.

(S)-Undec-1-yn-3-ol ((S)-5). Acetate $6(1.20 \mathrm{~g}, 5.71 \mathrm{mmol})$ was added to MeONa (1.50 g, $28 \mathrm{mmol})$ in anhydrous $\mathrm{MeOH}(20 \mathrm{~mL})$ and the mixture was stirred for $2 \mathrm{~h}$. The solvent was removed and $\mathrm{CH}_{2} \mathrm{Cl}_{2}$ $(10 \mathrm{~mL})$ and $2 \mathrm{~N} \mathrm{HCl}(10 \mathrm{~mL})$ were added. The layers were separated, the aqueous layer was extracted with $\mathrm{CH}_{2} \mathrm{Cl}_{2}(3 \times 5 \mathrm{~mL})$, the organic layer was dried over $\mathrm{MgSO}_{4}$ and solvents were removed to give $(S)$ $5(0.959 \mathrm{~g}, 5.70 \mathrm{mmol}, 100 \%)$ as a colorless oil: $[\alpha]_{\mathrm{D}}{ }^{25}+4.8\left(c 0.99, \mathrm{CHCl}_{3}\right)$.

The enantiomeric purity of the alcohol $\mathbf{5}$ was determined by HPLC analysis of the corresponding benzoate (13) prepared by reaction of 5 with benzoyl chloride. Racemic ester was separated into two peaks of $t_{\mathrm{R}} 6.3 \mathrm{~min}\left(R\right.$ enantiomer) and $7.3 \mathrm{~min}\left(S\right.$ enantiomer) employing a column CHIRALPAK ${ }^{\circledR}$ IA $(0.46 \mathrm{~cm} \varnothing \times 25 \mathrm{~cm})$ with hexane. The enantiomeric excess of $(S)-5$ was $98 \%$.

(S)-Undec-1-yn-3-yl benzoate (13). Colorless oil; $[\alpha]_{\mathrm{D}}^{25}-31.3\left(c 0.99, \mathrm{CHCl}_{3}\right) ; R_{f}$ (hexanes/AcOEt 8:2): 0.6; ${ }^{1} \mathrm{H} \mathrm{NMR}\left(\mathrm{CDCl}_{3}, 400 \mathrm{MHz}\right): \delta 0.88(3 \mathrm{H}, J=6.4 \mathrm{~Hz}), 1.25-1.40(10 \mathrm{H}, \mathrm{m}), 1.53(2 \mathrm{H}, \mathrm{q}, J=7.6$ 
Hz), $1.92(2 \mathrm{H}, \mathrm{m}), 2.48(1 \mathrm{H}, J=2.4 \mathrm{~Hz}), 5.59(1 \mathrm{H}, \mathrm{td}, J=6.8,2.0 \mathrm{~Hz}), 7.45(2 \mathrm{H}, \mathrm{m}), 7.57(1 \mathrm{H}, \mathrm{m}), 8.07$

$(2 \mathrm{H}, \mathrm{m}) ;{ }^{13} \mathrm{C} \mathrm{NMR}\left(\mathrm{CDCl}_{3}, 100 \mathrm{MHz}\right): \delta 14.1,22.6,24.9,29.1,29.2,29.4,31.8,34.7,64.4,73.6,81.3$, 128.4, 129.8, 129.9 133.1, 165.5; IR (film, $\mathrm{cm}^{-1}$ ): 3308, 3063, 2923, 2197, 1720, 1261; HRMS (ESI+): calcd for $\mathrm{C}_{18} \mathrm{H}_{25} \mathrm{O}_{2}(\mathrm{M}+\mathrm{H})^{+} 273.1849$, found 273.1844 .

(S)-Dodeca-1,2-dien-4-ol (4). A solution of dicyclohexylamine (2.23 mL, $11.3 \mathrm{mmol})$ and $(S)-5$ $(0.95 \mathrm{~g}, 5.6 \mathrm{mmol})$ in anhydrous dioxane $(20 \mathrm{~mL})$ was added dropwise under $\mathrm{N}_{2}$ to a stirred solution of paraformaldehyde $(0.42 \mathrm{~g}, 14.1 \mathrm{mmol})$ and $\mathrm{CuI}(0.538 \mathrm{~g}, 2.82 \mathrm{mmol})$ in anhydrous dioxane $(20 \mathrm{~mL})$. The mixture was heated at reflux for 4 hours. Solvent removal followed by flash chromatography (silica gel, hexanes/AcOEt 98:2) gave $4(0.885 \mathrm{~g}, 4.8 \mathrm{mmol}, 86 \%)$ as a yellow oil; $R_{f}$ (hexanes/AcOEt 8:2): $0.5 ;[\alpha]_{\mathrm{D}}{ }^{25}+2.6\left(c 0.99, \mathrm{CHCl}_{3}\right) ;{ }^{1} \mathrm{H} \mathrm{NMR}\left(\mathrm{CDCl}_{3}, 400 \mathrm{MHz}\right): \delta 0.86(3 \mathrm{H}, \mathrm{t}, J=6.4 \mathrm{~Hz}), 1.25-1.48(12 \mathrm{H}$, $\mathrm{m}), 1.54-1.60(3 \mathrm{H}, \mathrm{m}), 4.16(1 \mathrm{H}, \mathrm{m}), 4.85(2 \mathrm{H}, \mathrm{m}), 5.24(1 \mathrm{H}, \mathrm{q}, J=6.4 \mathrm{~Hz}) ;{ }^{13} \mathrm{C} \mathrm{NMR}\left(\mathrm{CDCl}_{3}, 100\right.$ MHz): $\delta 14.1,22.6,25.4,29.2,29.5,29.5,31.8,37.5,69.7,77.4,94.9,207.0 ;$ IR (film, $\left.\mathrm{cm}^{-1}\right): 3334$, 2921, 1955, 1035; HRMS (ESI+) calcd for $\mathrm{C}_{12} \mathrm{H}_{23} \mathrm{O}(\mathrm{M}+\mathrm{H})^{+}$183.1743, found 183.1742.

(S)-4-tert-Butyldimethylsilyloxydodeca-1,2-diene (7). A solution of tert-butyldimethylsilyl chloride $(1.10 \mathrm{~g}, 7.3 \mathrm{mmol})$ in anhydrous THF $(15 \mathrm{~mL})$ was added dropwise under $\mathrm{N}_{2}$ to a stirred solution of 4 $(0.665 \mathrm{~g}, 3.6 \mathrm{mmol})$, imidazole $(0.62 \mathrm{~g}, 9.0 \mathrm{mmol})$ at $\mathrm{rt}$. The mixture was stirred for $3 \mathrm{~h}$ and then quenched with saturated aqueous $\mathrm{NH}_{4} \mathrm{Cl}(10 \mathrm{~mL})$. The layers were separated, the aqueous layer was extracted with $\mathrm{CH}_{2} \mathrm{Cl}_{2}(3 \times 10 \mathrm{~mL})$, and the combined organic extracts were dried over $\mathrm{Mg}_{2} \mathrm{SO}_{4}$. Filtration, followed by solvent removal and chromatography (silica gel, hexanes/AcOEt 98:2) gave 7 $(0.962 \mathrm{~g}, 3.2 \mathrm{mmol}, 89 \%)$ as a colorless oil; $R_{f}$ (hexanes/AcOEt 95:5): 0.9; $[\alpha]_{\mathrm{D}}^{25}-9.6\left(c 0.99, \mathrm{CHCl}_{3}\right)$; ${ }^{1} \mathrm{H}$ NMR $\left(\mathrm{CDCl}_{3}, 400 \mathrm{MHz}\right): \delta 0.05(3 \mathrm{H}, \mathrm{s}), 0.06(3 \mathrm{H}, \mathrm{s}), 0.88-0.92(12 \mathrm{H}, \mathrm{m}), 1.24-1.40(12 \mathrm{H}, \mathrm{m}), 1.47-$ $1.57(2 \mathrm{H}, \mathrm{m}), 4.14(1 \mathrm{H}, \mathrm{m}), 4.72(2 \mathrm{H}, \mathrm{m}), 5.09(1 \mathrm{H}, \mathrm{q}, J=6.6 \mathrm{~Hz}) ;{ }^{13} \mathrm{C} \mathrm{NMR}\left(\mathrm{CDCl}_{3}, 100 \mathrm{MHz}\right):-4.9,-$ $4.3,14.1,18.2,22.7,25.5,25.9,29.3,29.5,29.6,31.9,38.7,71.6,75.7,95.0,207.4 ;$ IR $\left(\right.$ film, $\left.\mathrm{cm}^{-1}\right)$ : 2925, 1956, 1078; HRMS (ESI+) calcd for $\mathrm{C}_{18} \mathrm{H}_{37} \mathrm{OSi}(\mathrm{M}+\mathrm{H})^{+} 297.2608$, found 297.2599.

$(1 R, 2 S, 3 S)-3-t e r t-B u t y l d i m e t h y l s i l y l o x y-1-(t h i o p h e n-2-y l)-2-v i n y l u n d e c a n-1-o l \quad(s y n, a n t i-8) . \quad A$ solution of $7(0.962 \mathrm{~g}, 3.2 \mathrm{mmol})$ and 2-thiophenecarboxaldehyde $(0.36 \mathrm{~mL}, 3.9 \mathrm{mmol})$ in anhydrous 
$\mathrm{CH}_{2} \mathrm{Cl}_{2}(10 \mathrm{~mL})$ was added dropwise to a stirred suspension of dicyclohexylborane $(0.807 \mathrm{~g}, 4.5 \mathrm{mmol})$ in anhydrous $\mathrm{CH}_{2} \mathrm{Cl}_{2}(10 \mathrm{~mL})$ at $0{ }^{\circ} \mathrm{C}$ under $\mathrm{N}_{2}$. After $10 \mathrm{~min}$ at $0{ }^{\circ} \mathrm{C}$ the mixture was allowed to come to $\mathrm{rt}$ and was stirred for 4 hours, until it became homogeneous. Triethanolamine $(1.01 \mathrm{~mL}, 8.1 \mathrm{mmol})$ in $\mathrm{CH}_{2} \mathrm{Cl}_{2}(10 \mathrm{~mL})$ was added and stirring was continued for 1 hour. Solvent removal followed by flash chromatography (silica gel, hexanes/AcOEt 99:1) afforded syn,anti-8 as colorless oil (1.06 g, 2.6 mmol, $80 \%) ; R_{f}$ (hexanes/AcOEt 95:5): 0.3; $[\alpha]_{\mathrm{D}}{ }^{25}-4.4\left(c 0.99, \mathrm{CHCl}_{3}\right) ;{ }^{1} \mathrm{H} \mathrm{NMR}\left(\mathrm{CDCl}_{3}, 400 \mathrm{MHz}\right): \delta 0.12$ $(3 \mathrm{H}, \mathrm{s}), 0.18(3 \mathrm{H}, \mathrm{s}), 0.89(3 \mathrm{H}, \mathrm{t}, J=7.2 \mathrm{~Hz}), 0.95(9 \mathrm{H}, \mathrm{s}), 1.25-1.35(10 \mathrm{H}, \mathrm{m}), 1.35-1.45(2 \mathrm{H}, \mathrm{m}), 1.55-$ $1.65(2 \mathrm{H}, \mathrm{m}), 2.59(1 \mathrm{H}, \mathrm{td}, J=9.0,2.7 \mathrm{~Hz}), 3.99(1 \mathrm{H}, \mathrm{ddd}, J=7.8,5.6,2.7 \mathrm{~Hz}), 4.34(1 \mathrm{H}, \mathrm{d}, J=1.0 \mathrm{~Hz})$, $4.92(1 \mathrm{H}, \mathrm{ddd}, J=17.2,1.6,0.4 \mathrm{~Hz}), 5.01(1 \mathrm{H}, \mathrm{dd}, J=10.4,1.6 \mathrm{~Hz}), 5.09(1 \mathrm{H}, \mathrm{dd}, J=9.0,2.0 \mathrm{~Hz}), 5.60$ $(1 \mathrm{H}, \mathrm{ddd}, J=17.2,10.4,9.2 \mathrm{~Hz}), 6.90(2 \mathrm{H}, \mathrm{m}), 6.21(1 \mathrm{H}, \mathrm{dd}, J=4.8,1.6 \mathrm{~Hz}) ;{ }^{13} \mathrm{C} \mathrm{NMR}\left(\mathrm{CDCl}_{3}, 100\right.$ $\mathrm{MHz}): \delta-4.4,-4.3,14.1,18.0,22.7,25.9,26.1,29.2,29.5,29.6,31.8,33.0,56.2,71.5,75.5,118.4$, 124.1, 124.5, 126.1, 134.8, 148.3; IR (film, $\mathrm{cm}^{-1}$ ): 3446, 3073, 2926, 1252; HRMS (ESI+): calcd for $\mathrm{C}_{23} \mathrm{H}_{42} \mathrm{NaO}_{2} \mathrm{SSi}(\mathrm{M}+\mathrm{Na})^{+} 433.2567$, found 433.2564 .

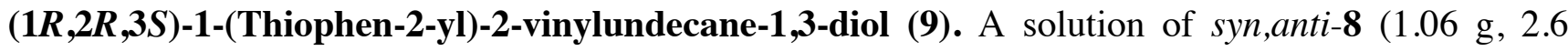
mmol) and $\mathrm{TBAF} \cdot 3 \mathrm{H}_{2} \mathrm{O}(4.07 \mathrm{~g}, 12.9 \mathrm{mmol})$ in anhydrous THF (15 mL) under $\mathrm{N}_{2}$ was stirred at rt for $24 \mathrm{~h}$. The mixture was quenched with a saturated aqueous $\mathrm{NH}_{4} \mathrm{Cl}(10 \mathrm{~mL})$ and the layers were separated. The aqueous layer was extracted with $\mathrm{CH}_{2} \mathrm{Cl}_{2}(3 \times 10 \mathrm{~mL})$ and the combined organic layers were dried over $\mathrm{MgSO}_{4}$. Filtration and solvent removal then gave the crude product that was purified by column chromatography (silica gel, hexanes/AcOEt 7:3) affording 9 as a colorless oil $0.642 \mathrm{~g}(2.2$ mmol, 84\%); $R_{f}$ (hexanes/AcOEt 8:2): 0.28; $[\alpha]_{\mathrm{D}}{ }^{25}-3.2\left(c 0.99, \mathrm{CHCl}_{3}\right) ;{ }^{1} \mathrm{H} \mathrm{NMR}\left(\mathrm{CDCl}_{3}, 400 \mathrm{MHz}\right): \delta$ $0.88(3 \mathrm{H}, \mathrm{t}, J=6.4 \mathrm{~Hz}), 1.23-1.35(12 \mathrm{H}, \mathrm{m}), 1.38-1.48(2 \mathrm{H}, \mathrm{m}), 2.31(1 \mathrm{H}, \mathrm{bs}), 2.48(1 \mathrm{H}, \mathrm{ddd}, J=8.8,6.4$, $2.0 \mathrm{~Hz}), 3.48(1 \mathrm{H}, \mathrm{bs}), 3.98(1 \mathrm{H}, \mathrm{m}), 5.10(1 \mathrm{H}, \mathrm{dd}, J=17.2,1.8 \mathrm{~Hz}), 5.13(1 \mathrm{H}, \mathrm{d}, J=6.2 \mathrm{~Hz}), 5.18(1 \mathrm{H}$, dd, $J=10.4,1.8 \mathrm{~Hz}), 5.89(1 \mathrm{H}, \mathrm{ddd}, J=17.2,10.4,9.2 \mathrm{~Hz}), 6.98(2 \mathrm{H}, \mathrm{m}), 7.24(1 \mathrm{H}, \mathrm{m}) ;{ }^{13} \mathrm{C} \mathrm{NMR}$ $\left(\mathrm{CDCl}_{3}, 100 \mathrm{MHz}\right): \delta 14.1,22.6,25.8,29.2,29.5,29.5,31.8,34.9,55.4,71.6,72.9,119.2,123.9,124.5$, 126.6, 134.1, 147.7; IR (film, $\mathrm{cm}^{-1}$ ): 3337, 3073, 2924, 1027; HRMS (ESI+): calcd for $\mathrm{C}_{17} \mathrm{H}_{28} \mathrm{NaO}_{2} \mathrm{~S}$ 
$(\mathrm{M}+\mathrm{Na})^{+} 319.1702$, found 319.1714

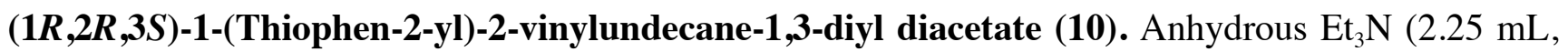
$16.5 \mathrm{mmol}), \mathrm{Ac}_{2} \mathrm{O}(1.55 \mathrm{~mL}, 16.5 \mathrm{mmol})$ and 4-DMAP (catalytic amount) were added to a solution of 9 (0.642 $\mathrm{g}, 2.2 \mathrm{mmol})$ in anhydrous $\mathrm{CH}_{2} \mathrm{Cl}_{2}(10 \mathrm{~mL})$ under $\mathrm{N}_{2}$. The reaction was stirred for 2 hours. $2 \mathrm{~N}$ $\mathrm{HCl}(10 \mathrm{~mL})$ was added and the aqueous layer was extracted with $\mathrm{CH}_{2} \mathrm{Cl}_{2}(3 \times 10 \mathrm{~mL})$. The organic layer was washed with $1 \mathrm{~N} \mathrm{NaOH}(10 \mathrm{~mL})$, dried over $\mathrm{MgSO}_{4}$, filtered and concentrated under vacuum to yield 10 as a colorless oil $(0.799 \mathrm{~g} 2.1 \mathrm{mmol}, 95 \%) ; R_{f}$ (hexanes/AcOEt 9:1): $0.88 ;[\alpha]_{\mathrm{D}}^{25}+9.7(c 0.99$, $\left.\mathrm{CHCl}_{3}\right) ;{ }^{1} \mathrm{H} \mathrm{NMR}\left(\mathrm{CDCl}_{3}, 400 \mathrm{MHz}\right): \delta 0.87(3 \mathrm{H}, \mathrm{t}, J=6.4 \mathrm{~Hz}), 1.23-1.35(12 \mathrm{H}, \mathrm{m}), 1.42-1.62(2 \mathrm{H}, \mathrm{m})$, $2.03(3 \mathrm{H}, \mathrm{s}), 2.04(3 \mathrm{H}, \mathrm{s}), 2.75(1 \mathrm{H}, \mathrm{td}, J=10.0,2.4 \mathrm{~Hz}), 4.94(1 \mathrm{H}, \mathrm{ddd}, J=17.2,1.7 \mathrm{~Hz}), 5.11(1 \mathrm{H}, \mathrm{dd}$, $J=10.2,1.7 \mathrm{~Hz}), 5.29(1 \mathrm{H}, \mathrm{ddd}, J=8.8,2.4,1.6 \mathrm{~Hz}), 5.60(1 \mathrm{H}, \mathrm{dt}, J=17.2,10.2 \mathrm{~Hz}), 5.97(1 \mathrm{H}, \mathrm{d}, J=10.2$ $\mathrm{Hz}), 6.90(1 \mathrm{H}, \mathrm{m}), 6.99(1 \mathrm{H}, \mathrm{m}), 7.23(1 \mathrm{H}, \mathrm{m}) ;{ }^{13} \mathrm{C} \mathrm{NMR}\left(\mathrm{CDCl}_{3}, 100 \mathrm{MHz}\right): \delta$ 14.1, 21.0, 21.0, 22.6, $25.3,29.2,29.4,29.5,31.8,32.7,53.0,69.5,71.3,121.1,125.5,126.3,127.0,131.9,142.1,170.0$ 170.6; IR (film, $\mathrm{cm}^{-1}$ ): 3076, 2925, 1740, 1237; HRMS (ESI+): calcd for $\mathrm{C}_{21} \mathrm{H}_{32} \mathrm{NaO}_{4} \mathrm{~S}(\mathrm{M}+\mathrm{Na})^{+}$ 403.1914, found 403.1920.

$(1 R, 2 R, 3 S)-2-(2-h y d r o x y e t h y l)-1-(t h i o p h e n-2-y l)$ undecane-1,3-diyl diacetate (11). A solution of 10 $(0.300 \mathrm{~g}, 0.78 \mathrm{mmol})$ in anhydrous $\mathrm{CH}_{2} \mathrm{Cl}_{2}(10 \mathrm{~mL})$ was added dropwise to a stirred suspension of dicyclohexylborane $(0.297 \mathrm{~g}, 1.7 \mathrm{mmol})$ in anhydrous $\mathrm{CH}_{2} \mathrm{Cl}_{2}(3 \mathrm{~mL})$ at $0{ }^{\circ} \mathrm{C}$, in a dry flask under $\mathrm{N}_{2}$. After 10 min at $0{ }^{\circ} \mathrm{C}$ the reaction was allowed to warm to $\mathrm{rt}$ and the mixture was stirred for 4 hours. A solution of $\mathrm{H}_{2} \mathrm{O}_{2}(1.5 \mathrm{~mL}, 33 \%)$ and phosphate buffer $(1.5 \mathrm{~mL}, \mathrm{pH}=7)$ was added and the mixture was stirred for $2 \mathrm{~h}$. The volatiles were removed under vacuum and purification by column chromatography (silica gel, hexanes/AcOEt 85:15) afforded product 11 as a colorless oil $(0.270 \mathrm{~g}, 0,68 \mathrm{mmol}, 87 \%) ; R_{f}$ (hexanes/AcOEt 85:15): 0.5; $[\alpha]_{\mathrm{D}}{ }^{25}+22.1\left(c 0.99, \mathrm{CHCl}_{3}\right) ;{ }^{1} \mathrm{H} \mathrm{NMR}\left(\mathrm{CDCl}_{3}, 400 \mathrm{MHz}\right): \delta 0.88(3 \mathrm{H}, \mathrm{t}$, $J=6.4 \mathrm{~Hz}), 1.23-1.35(12 \mathrm{H}, \mathrm{m}), 1.50-1.60(3 \mathrm{H}, \mathrm{m}), 1.69-1.77(1 \mathrm{H}, \mathrm{m}), 2.03(3 \mathrm{H}, \mathrm{s}), 2.04(3 \mathrm{H}, \mathrm{s}), 2.23$ $(1 \mathrm{H}, \mathrm{dtd}, J=10.0,4.8,2.4 \mathrm{~Hz}), 3.47(2 \mathrm{H}, \mathrm{m}), 5.26(1 \mathrm{H}, \mathrm{ddd}, J=8.0,5.6,2.4 \mathrm{~Hz}), 5.94(1 \mathrm{H}, \mathrm{d}, J=10.0 \mathrm{~Hz})$, $6.95(1 \mathrm{H}, \mathrm{m}), 7.07(1 \mathrm{H}, \mathrm{m}), 7.27(1 \mathrm{H}, \mathrm{m}) ;{ }^{13} \mathrm{C} \mathrm{NMR}\left(\mathrm{CDCl}_{3}, 100 \mathrm{MHz}\right): \delta 14.1,21.1,25.8,29.1,29.2$, 
29.4, 29.4, 31.8, 32.1, 43.4, 60.8, 70.8, 72.5, 125.7, 126.6, 127.0, 142.1, 167.0, 170.7; IR (film, cm ${ }^{-1}$ ):

3467, 3075, 2923, 1736, 1235; HRMS (ESI+): calcd for $\mathrm{C}_{21} \mathrm{H}_{34} \mathrm{NaO}_{5} \mathrm{~S}(\mathrm{M}+\mathrm{Na})^{+} 421.2019$, found 421.205.

$(2 R, 3 R)$-2-Acetoxy-3-[(S)-1-acetoxynonyl]pentanedioic acid (12). Ruthenium (III) chloride monohydrate $(5 \mathrm{mg}, 0.0197 \mathrm{mmol})$ was added to a solution of $11(0.147 \mathrm{~g}, 0.39 \mathrm{mmol})$ and $\mathrm{NaIO}_{4}$ (0.760 g, $3.55 \mathrm{mmol})$ in $\mathrm{CCl}_{4}(3 \mathrm{~mL}), \mathrm{CH}_{3} \mathrm{CN}(3 \mathrm{~mL})$ and $\mathrm{H}_{2} \mathrm{O}(4 \mathrm{~mL})$ and the mixture was stirred vigorously until TLC showed complete conversion. A saturated aqueous solution of $\mathrm{Na}_{2} \mathrm{CO}_{3}(5 \mathrm{~mL})$ was added and the layers were separated. The aqueous layer was acidified with $\mathrm{HCl} 1 \mathrm{~N}$ to $\mathrm{pH}=2$ and was extracted with AcOEt $(3 \times 5 \mathrm{~mL})$. The combined organic layers were dried over $\mathrm{MgSO}_{4}$, filtered and concentrated under vacuum to yield $\mathbf{1 2}$ as a colorless oil $(0.121 \mathrm{~g}, 0.323 \mathrm{mmol}, 82 \%) ; R_{f}$ $\left(\mathrm{CH}_{2} \mathrm{Cl}_{2} / \mathrm{MeOH}\right.$ 9:1): 0.1; ${ }^{1} \mathrm{H} \mathrm{NMR}\left(\mathrm{CD}_{3} \mathrm{OD} 400 \mathrm{MHz}\right): \delta 0.89(3 \mathrm{H}, \mathrm{t}, J=7.2 \mathrm{~Hz}), 1.23-1.35(12 \mathrm{H}, \mathrm{m})$, $1.55-1.69(2 \mathrm{H}, \mathrm{m}), 2.03(3 \mathrm{H}, \mathrm{s}), 2.11(3 \mathrm{H}, \mathrm{s}), 2.49(2 \mathrm{H}, \mathrm{d}, J=6.4 \mathrm{~Hz}), 2.81(1 \mathrm{H}, \mathrm{q}, J=6.4 \mathrm{~Hz}), 5.04(1 \mathrm{H}$, d, $J=5.2 \mathrm{~Hz}), 5.13(1 \mathrm{H}, \mathrm{q}, J=5.2, \mathrm{~Hz}) ;{ }^{13} \mathrm{C} \mathrm{NMR}\left(\mathrm{CD}_{3} \mathrm{OD}, 100 \mathrm{MHz}\right): \delta 14.5,20.6,20.9,23.8,26.6$, 30.4, 30.3, 30.6, 32.2, 33.1, 33.3, 40.9, 73.7, 74.1, 171.9, 172.2, 172.3, 175.6; IR (film, $\left.\mathrm{cm}^{-1}\right)$ : 33002500, 2921, 1737, 1702, 1248; HRMS (ESI+): calcd for $\mathrm{C}_{18} \mathrm{H}_{30} \mathrm{NaO}_{8}(\mathrm{M}+\mathrm{Na})^{+}$397.1833, found 397.1841.

(3aR,4S,6aR)-4-Octyldihydrofuro[3,4-b]furan-2,6(3H,6aH)-dione (2). $1 \mathrm{~N} \mathrm{H}_{2} \mathrm{SO}_{4}(2 \mathrm{~mL})$ was added to acid $12(0.070 \mathrm{~g}, 0.19 \mathrm{mmol})$ in dioxane $(4 \mathrm{~mL})$ and the mixture was heated at reflux for $24 \mathrm{~h}$. After cooling the solvents were removed and $\mathrm{CH}_{2} \mathrm{Cl}_{2}(5 \mathrm{~mL})$ and saturated aqueous $\mathrm{Na}_{2} \mathrm{CO}_{3}(5 \mathrm{~mL})$ were added. After stirring for 30 min the layers were separated and the aqueous layer was extracted with $\mathrm{CH}_{2} \mathrm{Cl}_{2}(3 \times 5 \mathrm{~mL})$. The organic combined organic extracts were dried over $\mathrm{MgSO}_{4}$ and the solvents were removed. Chromatographic purification (silica gel, $\mathrm{CH}_{2} \mathrm{Cl}_{2} / \mathrm{MeOH} 99: 1$ ) gave 2 as a colorless solid (0.036 g, $0.14 \mathrm{mmol}, 71 \%) ; R_{f}\left(\mathrm{CH}_{2} \mathrm{Cl}_{2} / \mathrm{MeOH}\right.$ 98:2): 0.9; $[\alpha]_{\mathrm{D}}^{25}-8.6\left(c 0.99, \mathrm{CHCl}_{3}\right) ;{ }^{1} \mathrm{H} \mathrm{NMR}\left(\mathrm{CDCl}_{3}\right.$, $400 \mathrm{MHz}): \delta 0.88(3 \mathrm{H}, \mathrm{t}, J=6.8 \mathrm{~Hz}), 1.24-1.37(10 \mathrm{H}, \mathrm{m}), 1.47-1.59(3 \mathrm{H}, \mathrm{m}), 1.78-1.86(1 \mathrm{H}, \mathrm{m}), 2.63$ $(2 \mathrm{H}, \mathrm{d}, J=9.6 \mathrm{~Hz}), 3.46(1 \mathrm{H}, \mathrm{qd}, J=9.6,5.8 \mathrm{~Hz}), 4.60(1 \mathrm{H}, \mathrm{td}, J=8.4,5.8 \mathrm{~Hz}), 5.14(1 \mathrm{H}, \mathrm{d}, J=8.3 \mathrm{~Hz}) ;{ }^{13} \mathrm{C}$ 
$\operatorname{NMR}\left(\mathrm{CDCl}_{3}, 100 \mathrm{MHz}\right): \delta 14.1,22.6,25.4,26.8,29.1,29.2,29.3,31.4,31.7,39.4,76.9,78.7,170.5$, 173.6; IR (film, cm ${ }^{-1}$ ): 2914, 2847, 1780, 1733; HRMS (ESI+): calcd for $\mathrm{C}_{14} \mathrm{H}_{26} \mathrm{NO}_{4}\left(\mathrm{M}+\mathrm{NH}_{4}\right)^{+}$ 272.1856, found 272.1851 .

(-)-Isoavenaciolide (-)-(1). Magnesium methyl carbonate (2.0 M in DMF, $3.5 \mathrm{~mL})$ was added to 2 $(0.045 \mathrm{~g}, 0.18 \mathrm{mmol})$ and the mixture was heated at $140^{\circ}$ for $6 \mathrm{~h}$ under $\mathrm{N}_{2}$. After cooling, the mixture was carefully added to cold, stirred $6 \mathrm{~N} \mathrm{HCl}(5 \mathrm{~mL})$ and $\mathrm{CH}_{2} \mathrm{Cl}_{2}$. When vigorous gas evolution had subsided the organic layer was separated and the aqueous layer was extracted with $\mathrm{CH}_{2} \mathrm{Cl}_{2}(4 \times 10 \mathrm{~mL})$. The combined organic extracts were washed with $\mathrm{H}_{2} \mathrm{O}(10 \mathrm{~mL})$, dried over $\mathrm{MgSO}_{4}$, and the solvents were removed. The residual yellow oil was treated with a solution of glacial acetic $(1 \mathrm{~mL})$, formalin $(1$ $\mathrm{mL}), N$-methylaniline $(0.5 \mathrm{~mL})$ and sodium acetate $(0.040 \mathrm{~g})$. The mixture was stirred vigorously for $2 \mathrm{~h}$ at $\mathrm{rt}$ and then was diluted with a mixture of saturated $\mathrm{NaCl}$ and conc $\mathrm{HCl}(5: 1)$ and $\mathrm{CH}_{2} \mathrm{Cl}_{2}(5 \mathrm{~mL})$ and the layers were separated. The aqueous layer was extracted with $\mathrm{CH}_{2} \mathrm{Cl}_{2}(3 \times 10 \mathrm{~mL})$ and the combined organic extracts were dried over $\mathrm{MgSO}_{4}$ and the solvents were removed. Purification by column chromatography (silica gel, hexanes/AcOEt 7:3) afforded of (-)-isoavenaciolide ((-)-1) (0.038 g, 0.14 mmol, $78 \%)$ as a white solid; mp 126-128 ${ }^{\circ} \mathrm{C} ; R_{f}$ (hexanes/AcOEt 8:2): 0.05; $[\alpha]_{\mathrm{D}}^{25}-153.9(c 0.99$, $\mathrm{EtOH}) ;{ }^{1} \mathrm{H} \mathrm{NMR}\left(\mathrm{CDCl}_{3}, 400 \mathrm{MHz}\right): \delta 0.88(3 \mathrm{H}, \mathrm{t}, J=6.4 \mathrm{~Hz}), 1.25-1.50(10 \mathrm{H}, \mathrm{m}), 1.52-1.71(4 \mathrm{H}, \mathrm{m})$, $3.99(1 \mathrm{H}, \mathrm{tt}, J=8.4,2.2 \mathrm{~Hz}), 4.78(1 \mathrm{H}, \mathrm{ddd}, J=9.6,8.4,3.2 \mathrm{~Hz}), 5.10(1 \mathrm{H}, \mathrm{d}, J=8.4 \mathrm{~Hz}), 5.88(1 \mathrm{H}, \mathrm{d}$, $J=2.2 \mathrm{~Hz}), 6.61(1 \mathrm{H}, \mathrm{d}, J=2.2 \mathrm{~Hz}) ;{ }^{13} \mathrm{C} \mathrm{NMR}\left(\mathrm{CDCl}_{3}, 100 \mathrm{MHz}\right): \delta 14.1,22.6,26.0,29.1,29.1,29.3$, 31.8, 32.4, 41.7 , 74.7, 80.4, 128.9, 130.8, 167.8, 170.0; IR (film, $\left.\mathrm{cm}^{-1}\right): 3021,2932,2843,1793 ;$ HRMS (ESI+): calcd for $\mathrm{C}_{15} \mathrm{H}_{26} \mathrm{NO}_{4}\left(\mathrm{M}+\mathrm{NH}_{4}\right)^{+} 284.1856$, found 284.1854 .

ACKNOWLEDGMENT. This work was supported by the Spanish Ministerio de Educación y Ciencia (CTQ2006-13249 and CTQ2009-09692). We thank the Generalitat de Catalunya for a doctorate 
studentship to C.S. and the University of Barcelona for a fellowship to D.S. We also thank Anna Pou for assistance in the preparation of some starting materials. The authors are grateful to Novozymes Spain SA for a generous gift of Novozym 435.

\section{SUPPORTING INFORMATION}

${ }^{1} \mathrm{H}$ and ${ }^{13} \mathrm{C}$ NMR spectra of $\mathbf{1 , 2 , 4 - 1 3}$. This material is available free of charge via the Internet at http://pubs.acs.org.

\section{REFERENCES AND FOOTNOTES}

1. Aldridge, D. C.; Turner, W. B. J. Chem. Soc., C 1971, 2431-2432.

2. Ueda, K.; Usui, T.; Nakayama, H.; Ueki, M.; Takio, K.; Ubukata, M.; Osada, H.; FEBS Lett. 2002, $525,48-52$.

3. For a review, see: Martín, V. S.; Rodríguez, C. M.; Martín T. Org. Prep. Proced. Int. 1998, 30, 291-324.

4. (a) Ohrui, H.; Emoto, S. Tetrahedron Lett. 1975, 16, 3657-3660. (b) Anderson, R. C.; FraserReid, B. Tetrahedron Lett. 1977, 18, 2865-2868. (c) Anderson, R. C.; Fraser-Reid, B. J. Org. Chem. 1985, 50, 4781-4786. (d) Wee, A. G. H. Tetrahedron 1990, 46, 5065-5076 and correction: Wee, A. G. H. Tetrahedron 1993, 49, 1335. For formal syntheses, see: (e) McDonald, C. E.; Dugger, R. W. Tetrahedron Lett. 1988, 29, 2413-2416. (f) Chida, N.; Tobe, T.; Suwama, M.; Ohtsuka, M.; Ogawa, S. J. Chem. Soc., Chem. Commun. 1990, 994-995. (g) Chida, N.; Tobe, T.; Suwama, M.; Ohtsuka, M.; Ogawa, S. J. Chem. Soc., Perkin Trans. 1 1992, 2667-2673. (h) For the (+)-enantiomer, see: Alcázar, E.; Kassou, M.; Matheu, I.; Castillón, S. Eur. J. Org. Chem. 
2000, 2285-2289. (i) For other synthetic approaches, see: Al-Tel, T. H.; Al-Qawasmeh, R. A.; Sabri, S. S.; Voelter, W. J. Org. Chem. 2009, 74, 4690-4696 and references therein.

5. (a) Suzuki, K.; Miyazawa, M.; Shimazaki, M.; Tsuchihashi, G. Tetrahedron Lett. 1986, 27, 62376240. (b) Suzuki, K.; Miyazawa, M.; Shimazaki, M.; Tsuchihashi, G. Tetrahedron 1988, 44, 4061-4072. (c) Burke, S. D.; Pacofsky, G. J.; and Piscopio, A. D. J. Org. Chem. 1992, 57, 22282235. (d) Rodríguez, C. M.; Martín, V. S.; Martín T. J. Org. Chem. 1996, 61, 8448-8452. For formal syntheses, see: (e) Ito, K.; Fukuda, T.; Katsuki, T. Synlett 1997, 387-389. (f) Ito, K.; Fukuda, T.; Katsuki, T. Heterocycles 1997, 46, 401-411.

6. (a) Yu, C.-M.; Youn, J.; Jung, J. Angew. Chem. Int. Ed. 2006, 45, 1553-1556. (b) For a formal synthesis, see: Labeeuw, O.; Blanc, D.; Phansavath, P.; Ratovelomanana-Vidal, V.; Genêt, J.-P. Eur. J. Org. Chem. 2004, 2352-2358. (c) For a recent synthetic approach, see: Blot, V.; Reboul, V.; Metzner, P. Eur.J. Org. Chem. 2006, 1934-1939.

7. Sánchez, C.; Ariza, X.; Cornellà, J.; Farràs, J.; Garcia, J.; Ortiz, J. Chem. Eur. J. 2010, 16, 1153511538.

8. For the use of allenes in natural product syntheses, see: Yu, S.; Ma, S. Angew. Chem. Int. Ed. 2012, 51, 3074-3112.

9. (a) Ma, S.; Hou, H.; Zhao, S.; Wang, G. Synthesis 2002, 1643-1645. (b) Kuang, J.; Ma, S. J. Org. Chem. 2009, 74, 1763-1765.

10. (a) Raminelli, C.; Comasseto, J. V.; Andrade, L. H; Porto, A. L. M. Tetrahedron:Asymmetry 2004, 15,3117-3122. (b) Xu, D.; Li, Z.; Ma, S. Tetrahedron Lett. 2003, 44, 6343-6346.

11. Enantiomeric excess was determined by HPLC analysis of the benzoyl derivatives of $\mathbf{5}$.

12. The anti,anti isomer is obtained when an aliphatic aldehyde is used under these conditions. This 
relative stereochemistry results from the addition of the (Z)-2-alkenylborane to the opposite face of the aldehyde.

13. Relative stereochemistry was determined by analysis of ${ }^{1} \mathrm{H}$ NMR coupling constants (see supplementary material of reference 7). The syn relationship between $\mathrm{CHOTBS}$ and $\mathrm{CHCH}=\mathrm{CH}_{2}$ is usually characterized by a $J<3 \mathrm{~Hz}(J=2.0 \mathrm{~Hz}$ for compound 8 compared to $J>6 \mathrm{~Hz}$ for the anti relationship) whereas the anti relationship between $\mathrm{CHCH}=\mathrm{CH}_{2}$ and $\mathrm{CHAr}$ is usually characterized by a $J \approx 9-10 \mathrm{~Hz}(J=9.0 \mathrm{~Hz}$ for compound 8 compared to $J \approx 4-8 \mathrm{~Hz}$ for the syn relationship).

14. Carlsen, P. H. J.; Katsuki, T.; Martin, V. S.; Sharpless, K. B. J. Org. Chem. 1981, 46, 3936-3938.

15. Murta, M. M.; de Azevedo, M. B. M.; Greene, A. E. Synth. Commun. 1993, 23, 495-503.

SYNOPSIS TOC.

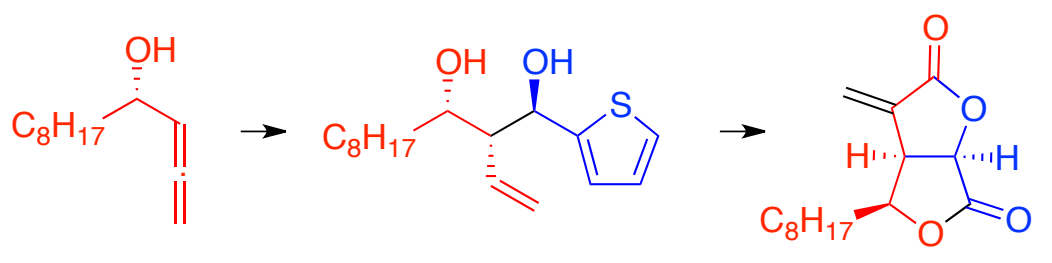

(-)-Isoavenaciolide 